\title{
MULTIPLE VISUALIZATION WEB APPROACH FOR CULTURAL HERITAGE OBJECTS
}

\author{
Dante ABATE ${ }^{1}$, Graziano FURINI ${ }^{1}$, Silvio MIGLIORI ${ }^{2}$, Samuele PIERATTINI ${ }^{1}$ \\ ${ }^{1}$ ENEA Research Centre, UTICT \\ Via Martiri di Montesole 4, Bologna 40129, Italy \\ \{dante.abate, graziano.furini, samuele.pierattini\}@enea.it \\ ${ }^{2}$ ENEA Headquarters, UTICT \\ Lungotevere Thaon de Revel 76, Rome 00196, Italy \\ silvio.migliori@enea.it
}

Keywords: laser scanning, 3D modeling, web visualization, HPC, QuickTime VR Object (QTVR)

\begin{abstract}
Usually the diffusion and sharing of cultural heritage documented 3D models on the web are not first of concern for scholars due to the fear of losing the intellectual property related to them. Sometimes the interaction and navigation of virtual objects via the World Wide Web is also problematic due to their dimension (number of triangles), when high-definition has to be preserved. In this paper we propose a mash up methodology, for a multiple approach to visualize 3D models over the internet. After the digitization of a marble statue placed in the Medieval Museum of the city of Bologna, according to the well known 3D pipeline (from the laser scan survey to the texturing process), we assembled together different solutions for sharing the model on the web.
\end{abstract}

\section{INTRODUCTION}

\subsection{Content}

Today it should be possible for an end-user to take advantage of web resources to view high-definition 3D models of cultural heritage artifacts and monuments. Ideally, this should be possible online and in real time without a browser plug-in. But, to date, this goal has not been reached. Nowadays, to distribute a high-definition 3D model on the network means giving the model itself to the user who needs to download the whole file, has sufficiently powerful hardware, a high speed connection, and installs, if available, the application for display, or reaches a compromise solution where the resolution during navigation and interaction with the virtual object is low and the rendering of the image is blurred and fuzzy [1,2]. However, different resources and techniques allow visualizing 3D models on the web. The solutions proposed here are suitable for different users and different goals.

\subsection{D Modeling}

The first step of the process has been the digitization of a statue, probably a base of an "Acquasantiera" (the basin where the holy water is usually stored), today placed in the Medieval Museum of the City of Bologna [3]. The equipment used was the Next Engine Desktop 3D Scanner mounted over a tripod (Figure 1). The scan data have been processed in Meshlab v.1.3.0 open source software developed by the Visit Computing Lab, ISTI, Italian National Research Council [4]. A wide range of filters were applied to clean and repair each range map. Afterward a semiautomatic approach was used for the alignment of all the sixty range maps in order to transform them in the same coordinate system using an ICP (Iterative Closest Point) algorithm. Finally the Poisson Surface Reconstruction was applied. The Poisson Surface Reconstruction can reconstruct high quality surfaces through a global optimization. The model was texturized using the UV modifier of 3D Studio Max 9 software. This method uses UV coordinates to attach texture to a model. After all the post-processing steps the 3D high-definition model of the "Acquasantiera" consists of 6 million triangles plus the texture information (Figure 2). Although it was possible to decimate the model significantly, the largest amount of triangles was preserved in order to save the high quality and details of the object. 


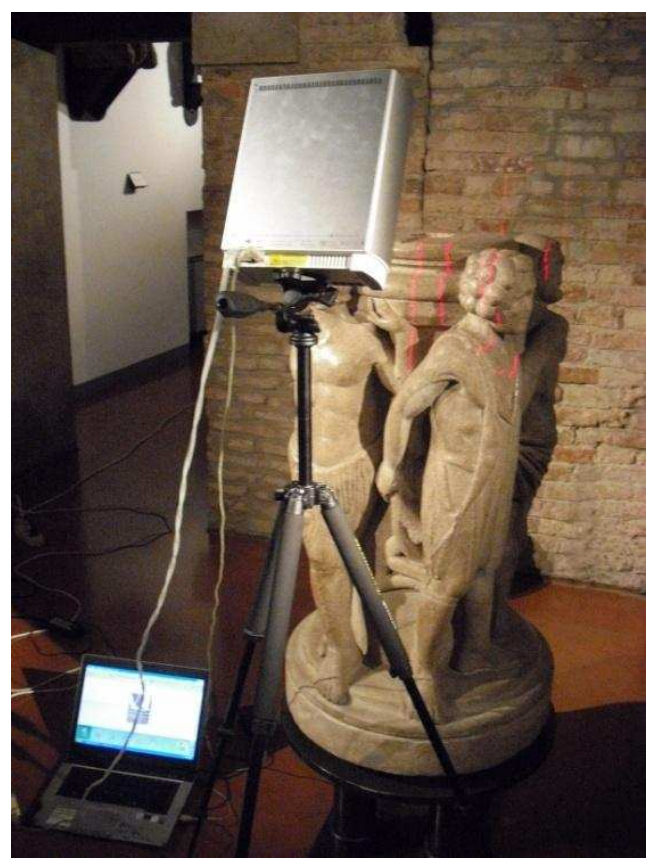

Figure 1: Laser Scanner Survey

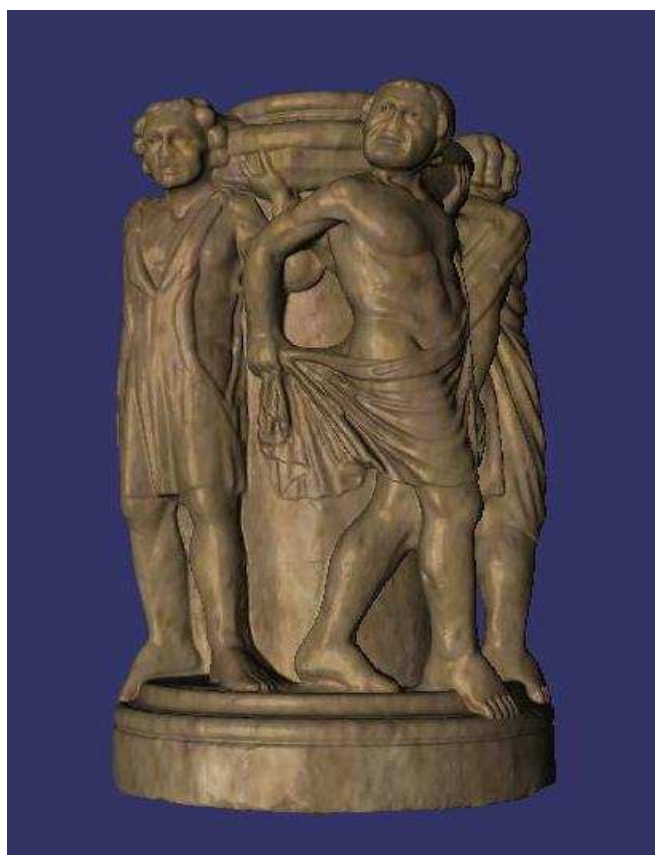

Figure 2: Virtual High-Definition 3D Model

\section{OUTPUT}

The solutions made available to the final user to visualize the model on the web are:

- High-Definition Remote Rendering on a HPC infrastructure. The technology developed for the remote visualization (ARK3D, the ENEA-GRID infrastructure for the Remote 3D) frees the user from the need of specific hardware and software resources, and protects the intellectual property related to the 3D model, since it is not downloaded locally. The user interacts with the virtual environment using the remote hardware and software resources [5].

- 3D No Plugins (3DNP) is a 3D viewer that doesn't need browser plug-in, only JavaScript [6]. It requires a series of images which can be acquired either through a digital camera or by software when the object has been previously digitized.

- QuickTime VR Object (QTVR). It allows the creation and viewing of photographically captured objects through images taken at multiple viewing angles. It functions for the standalone QuickTime Player, as well as for the QuickTime Web browser plug-in [7].

- Virtual Reality Mark Up Language (VRML) file format for model download.

\subsection{High Definition Remote Rendering on a HPC Infrastructure}

The first possibility made available to visualize the "Acquasantiera" on the World Wide Web was a remote rendering approach exploiting the ARK3D architecture. The study performed by ENEA, within the project CRESCO, concerns the implementation of a hardware and software architecture (ARK3D) that allows remote access to a repository of three-dimensional models (high resolution, multi-disciplinary, available via the internet, and provided and uploaded by registered users). The technology developed for the remote visualization frees the user from the need of specific hardware and software resources, and protects the intellectual property related to the $3 \mathrm{D}$ model, since it is not downloaded locally. The user interacts with the high-definition virtual environment using the remote hardware and software resources (Remote 3D) (Figure3). This project uses the ICT infrastructure of ENEA-GRID and in particular the graphic cluster built up for CRESCO project and available at the ENEA Centre of Portici (Napoli, Italy). The 3D models can be uploaded by users, due to registration and verification of the content. The database is queried via the web by free search keywords. The result will contain the textual data available, and a link to display a high-resolution threedimensional version of the model (3D Remote). The Remote 3D is done using a dedicated graphic cluster, which guarantees the protection of the data. The whole architecture is scalable, both in the number of models uploaded and in 
the number of simultaneous users logging in. The database is queried via a web page. The result contains all the available documentation on selected models. For non-registered users, together with the metadata, there will be a link to images (screenshots) of the model. For registered users, in addition to the documentation, there will be a link to run the remote applications which allows the visualization of three dimensional models. The display can be made through any graphics application, both proprietary and Open Source that uses OpenGL on Linux operating systems. Within this project the standard viewer was implemented using Open Scene Graph libraries [8]. In essence the application runs on the remote machine (server), using the hardware resources including the graphics card for rendering (Remote Rendering). Nowadays, the graphic cluster dedicated to the project consists of 4 workstations with AMD dual-core processors, 16GB of RAM and a NVidia Quadro FX. The user, through the dedicated web page, has to run a Java applet which installs and automatically configures the client to access the graphics application. The user is not required to know any configuration features or install other kinds of applications. Between the client and the server the data transfer is exclusively represented by a stream of compressed images, generated remotely by the application, together with the functions of keyboard and mouse interactions. Operations delegated to the client are limited to decompression of the images and keyboard and mouse input management. With this kind of technology the user can utilize hardware tools such as a netbook or PDA, even with the limited bandwidth connection, to access the platform.

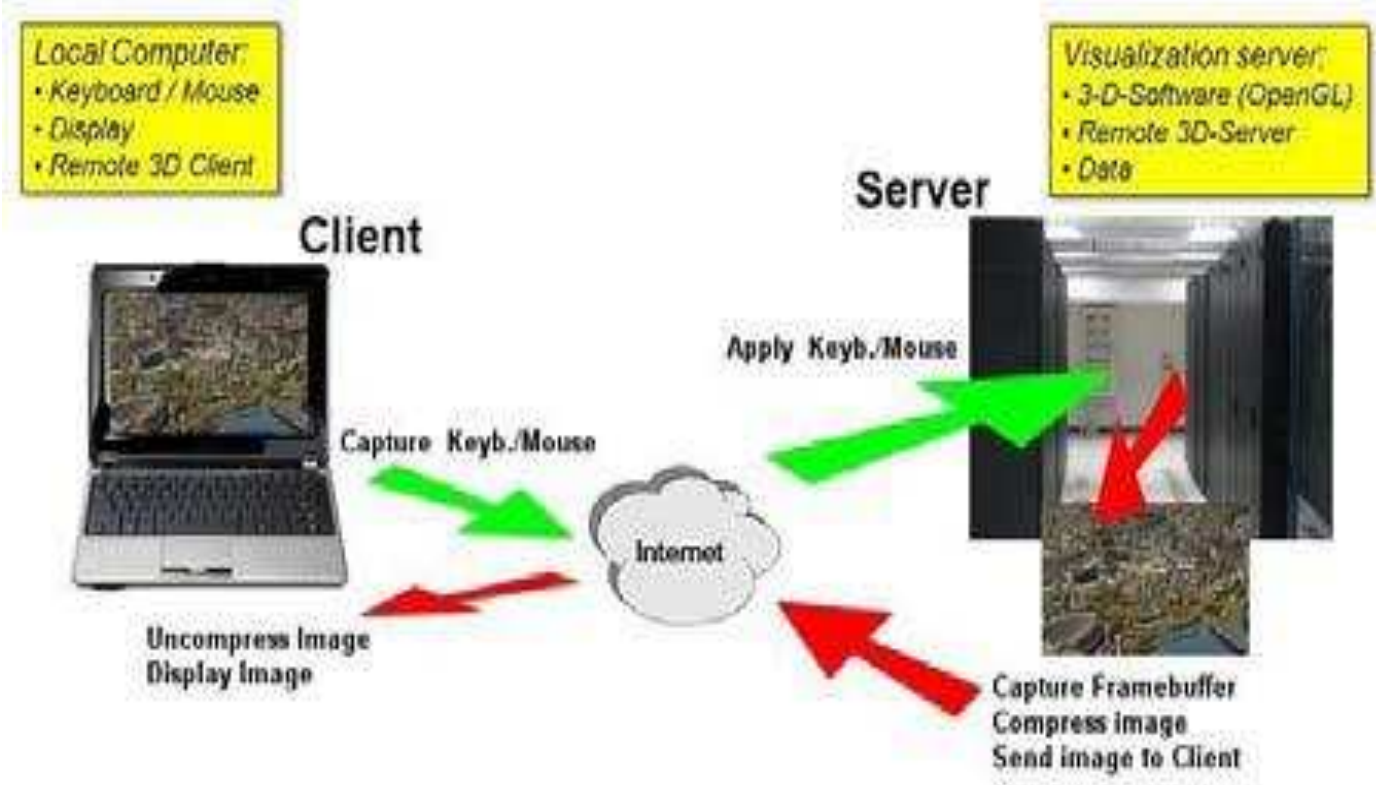

Figure 3: Remote 3D Rendering Based on CRESCO Graphic Section

\subsection{D No Plugins (3DNP)}

3D No Plugins is a java script able to upload an image series on a website and to simulate a 3D view, showing the appropriate image according to the user's mouse movement. 3DNP is licensed under GNU Public License (GPL) and is therefore completely free. In contrast with Panoramas pictures, which are captured from one location looking out at various angles, objects are captured from many locations pointing in toward the same central object. The simplest type of Object VRs to capture is single row, typically captured around the equator of an object. Capturing a multi-row object movie requires a more elaborate setup for capturing images, because the camera must be tilted above and below the equator of the object at several tilt angles. For this study the image series were obtained by exploiting a Python script for Blender, the open source 3D modeling software. This script is useful for quickly creating the images for the java script. Three hundred twenty-four images were rendered. Once the script is running, the 3D model previously created will appear inside a sphere formed by some vertices (Figure 4). These vertices represent the points where the Blender Camera will be virtually placed so it can render the model from different points of view. Four different parameters can be modified in order to set the virtual sphere around the 3D model.

Level: sets the horizontal subdivision

Degrees: sets the vertical subdivision

CameraBoss: define the size

CapBuffer: define how flat 
By increasing the "Level" and "Degrees" values the script will produce more rendered images. The result will be a more realistic and smooth interaction with the 3D model on the web site where the object will be embedded (Figure 5). Naturally more images will result in a larger computing time.
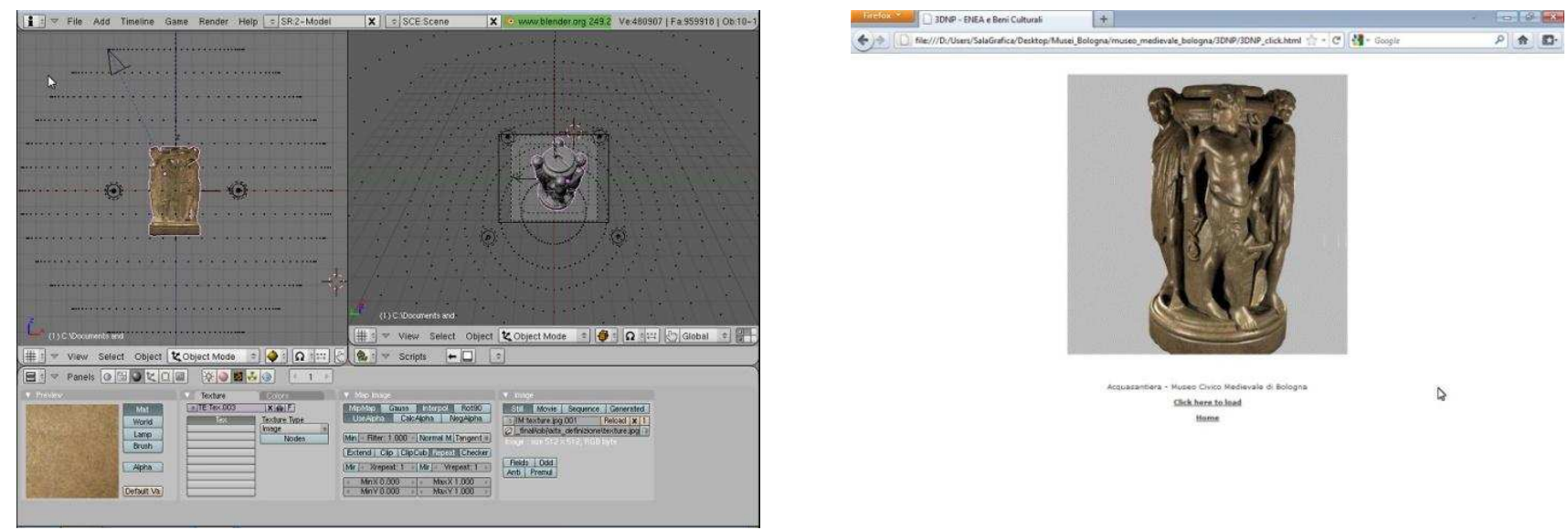

Figure 4: Python Script for Blender Interface

Figure 5: 3D No Plugins Web Page Embedded

\subsection{QuickTime VR Object (QTVR)}

The three hundred twenty-four images produced with the Python script for Blender were used to create interactive QuickTime VR Objects (QTVR Object). QuickTime Virtual Reality is an extension of the QuickTime technology developed by Apple Computer, Inc. that allows viewers to interactively explore and examine photo realistic, threedimensional objects and virtual worlds. While the 3D No Plugins will be embedded into the web page, the QuickTime object movie file (extension .mov) will be opened by Apple's Movie Player. One of the images will be displayed on the screen according to the position of the mouse cursor. Then, when moving the mouse, the images will change one after another continuously so as to give a movement like an animation (Figure 6).

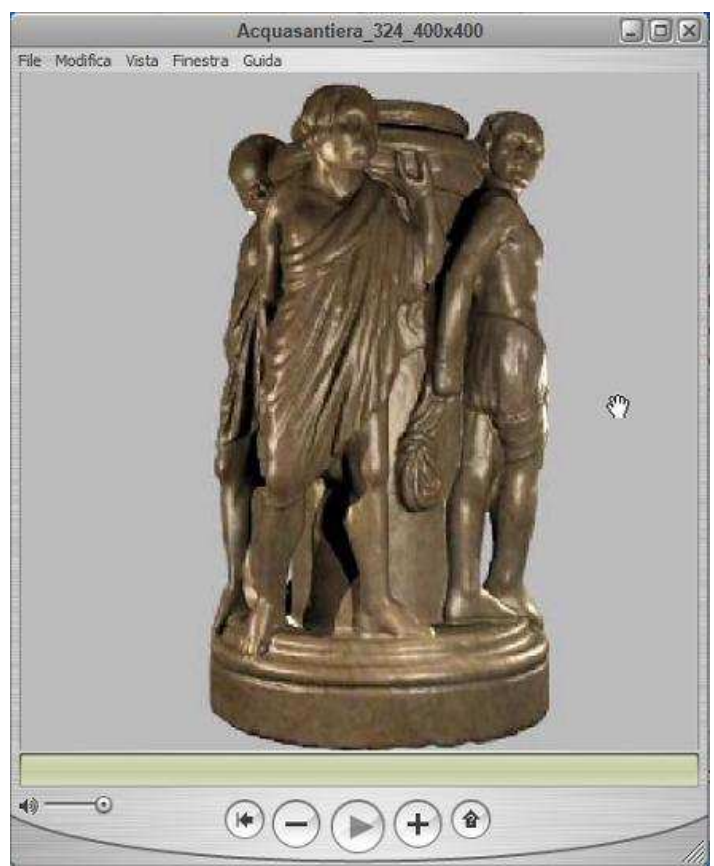

Figure 6: QuickTime Movie Player 
The viewer will interact and navigate the VR object using conventional computer input devices (such as the mouse, trackball, track pad or keyboard) to change the displayed image via the QuickTime VR movie controller. Together with the traditional orbit tool the user will be able to experience also the magnifier tool. However, an image too magnified could result in a loss of definition and quality. To create the QTVR object, the VR Worx suite has been used. The VR Worx gives you the ability to generate QTVR Panoramic movies, Object movies and Multi-node scenes.

\subsection{Downloadable File Formats}

Eventually the original 3D model was also converted in VRML file to allow the final user to visualize it through a standard VRML player locally with full interaction. Two VRML files, both with texture information attached, were created. A high-definition model of more than 6 million polygons (490 MB) and a low-definition model of 250 thousand polygons (32 MB).

However, this approach assumes:

- the necessity to download the 3D model itself from the web and consequently the need of a high bandwidth connection;

- the installation of a VRML viewer;

- the loss of control on the 3D model and on its intellectual rights by the former owner when external users get in possession of it.

\section{CONCLUSIONS}

The parameters intended to be preserved for this study were:

- real time interaction;

- definition/quality;

- $\quad$ intellectual rights attached to the model.

Aside from the remote rendering technique, all other approaches showed a lack in definition, interaction and/or intellectual rights preservation, separately or together.

Indeed, both the 3D No Plugins and QTVR object show a loss in:

- definition/quality;

- interaction;

- control over the model intellectual rights (the series of images will be downloaded on the user's pc cache).

On the other hand, locally downloading the complete 3D model (VRML) will guarantee full interaction and highdefinition even if the final user has to install the proper visualization software and own the needed hardware resources. Only the remote rendering approach on the HPC infrastructure (ARK3D) is able to preserve all the values together at once. In conclusion the choice of the correct visualization approach definitely belongs to the final goal of the user's project.

\section{REFERENCES}

[1] Koller. D, Turitzin M., Levoy M., Tarini M., Croccia G., Cignoni, P., Scopigno R., Protected Interactive 3D Graphics Via Remote Rendering, Proceedings of the 31st International Conference on Computer Graphics and interactive techniques, SIGGRAPH 2004, Los Angeles, August 8-12

[2] Koller D., Levoy M., Protecting 3D Graphics Content, Communications of the ACM, 48(6):74-80, June 2005

[3] Abate D., Ciavarella R., Furini G., Guarnieri G., Migliori S., Pierattini S., 3D modeling and remote rendering technique of a high definition cultural heritage artefact, Procedia Computer Science Volume3, 2011,Pages 848-852, World Conference on Information Technology

[4] http://meshlab.sourceforge.net/

[5] https://www.ark3d.enea.it/home.html

[6] http://www.thoro.de/page/3dnp-introduction-en

[7] http://www.apple.com/quicktime/download

[8] Abate D., Ciavarella R., Frischer B., Furini G., Guarnieri G., Migliori S., Pierattini S., 3DWS - 3D Web Service Project, Proceedings of Computer Applications and Quantitative Methods in Archaeology, CAA 2010, Granada, Spain 6-9 April 2010 (in printing). 